\title{
Effect of strain on $\mathrm{GaAs}_{1-x-y} \mathrm{~N}_{x} \mathrm{Bi}_{y} / \mathrm{GaAs}$ to extract the electronic band structure and optical gain by using 16-band $k p$ Hamiltonian
}

\author{
ARVIND SHARMA and T D DAS* \\ Department of Basic \& Applied Science, National Institute of Technology, Papum Pare 791112, Arunachal Pradesh, \\ India \\ *Author for correspondence (tddas@ hotmail.com)
}

MS received 12 April 2018; accepted 12 August 2018; published online 27 March 2019

\begin{abstract}
GaAs}_{1-x-y} \mathrm{~N}_{x} \mathrm{Bi}_{y}$ is a suitable candidate for $1.06 \mu \mathrm{m}$ solid state lasers and high-efficiency solar cells. Mathematical models such as 16-band $k p$ model is used to study the band structure, strain generated effect, band offset and variation of their parameters with $\mathrm{Bi}$ and $\mathrm{N}$ concentrations. Lattice constants of alloy $\mathrm{GaAs}_{1-x-y} \mathrm{~N}_{x} \mathrm{Bi}_{y}$ with $x / y=0.58$ can match those of GaAs with the incorporation of $\mathrm{Bi}$ and $\mathrm{N}$ into GaAsNBi. Arsenic atom substitution due to the incorporation of $\mathrm{N}$ and $\mathrm{Bi}$ impurity atoms causes a significant band gap reduction of $\sim 200 \mathrm{meV}$ for $\mathrm{GaAs}_{0.937} \mathrm{~N}_{0.023} \mathrm{Bi}_{0.04}$ alloys under lattice-matched conditions and in addition, by tuning the concentrations of $\mathrm{N}$ and $\mathrm{Bi}$, the electrical and optical properties of GaAsNBi can be controlled. Optical gain of $\mathrm{GaAs}_{1-x-y} \mathrm{~N}_{x} \mathrm{Bi}_{y}$ quantum well $(\mathrm{QW})$ and $\mathrm{GaAs}$ as a barrier are calculated in generalized mode and observed the effect of the energy level of GaAs barrier on the GaAsNBi QW.
\end{abstract}

Keywords. $\mathrm{GaAs}_{1-x-y} \mathrm{~N}_{x} \mathrm{Bi}_{y} ; k p$ method; strain; optical gain.

\section{Introduction}

During the last few decades, III-V-Bi/N alloys have attracted attention towards research because of good physical properties. The coalloying of $\mathrm{Bi}$ and $\mathrm{N}$ in GaAs provides application in optoelectronics and makes them attractive candidates for the design of highly capable mid- and far-infrared optical and spintronics devices, providing large and resilient parameter space for band structure engineering [1]. $\mathrm{GaAs}_{1-x-y} \mathrm{~N}_{x} \mathrm{Bi}_{y}$ is proposed as quaternary alloys to fabricate new devices in optoelectronic fields, such as $1.06 \mu \mathrm{m}$ solid-state lasers and high-efficiency multi-junction solar cells, and became important to study optical and electronic properties [2]. GaAsN has been studied in the past few decades because of the large bowing parameter and nitrogen is known to be responsible for structural and electronic defects that effect on optoelectronic efficiency, but it decreases the band gap and lattice constants; obtaining high-quality GaAs GaAsN/GaAs is difficult where as co-alloying with Bi effectively matched the lattice constant of GaAs [3]. A lower value of energy band gap is attainable with GaAsNBi with lattice constant matched with GaAs by changing the concentrations of $\mathrm{N}$ and $\mathrm{Bi}$. The corresponding band gap reductions are due to resonant interactions rather than alloying. In the case of $\mathrm{N}$, an isolated state resonant with the conduction band of GaAs is imported, while Bi imported a state resonant with the valence band which leads to increase in the carrier concentration without change in mobility [4]. In addition, incorporation of $\mathrm{N}$ and $\mathrm{Bi}$ into $\mathrm{GaAs}$ introduced the compressive and tensile strains. Strong perturbation for Bi concentration for low value of strain auger-suppressing band structure condition satisfied $E_{\mathrm{g}}<\Delta_{\mathrm{SO}+}$ [5]. Tight banding model (TB), semi-empirical methods, band anticrossing (BAC) theory and density functional theory (DFT) are effective to focus on theoretical simulations of structural and electronic properties of III-V dilute $\mathrm{Bi}$ and $\mathrm{N}$ containing structure including surfaces, thin films, nanostructures and clusters. Among those reliable computational methods, the most popular one is the $k p$ model. The $k p$ model provides simple and accurate descriptions of the band structure of dilute $\mathrm{Bi}$ and $\mathrm{N}$ alloys of GaAs in the vicinity of the band edges and hence, will be of importance for the modelling and design of future III-V dilute $\mathrm{N}$ - and Bi-based optoelectronic devices. Here, we have studied the 16-band $\mathrm{kp}$ model to obtain the band structure and to observe the effect on energy with different mole fractions of $\mathrm{N}$ and $\mathrm{Bi}$ in $\mathrm{GaAs}_{1-x-y} \mathrm{~N}_{x} \mathrm{Bi}_{y}$ for a strained layer. In addition, the $k p$ method is widely used to describe band structure of bulk and nanostructures of semiconductors near the $\Gamma$ point and the band parameters, such as the band gap, band offsets, spin-orbit (SO) splitting energy and strain of GaAsBiN alloys on GaAs have been reported [5,6]. For the first time Yoshimoto et al [7] experimentally have grown successfully GaNAsBi by molecular beam epitaxy with a Bi content of $4 \%$ and an $\mathrm{N}$ content of $8 \%$ in 2004. The highest $\mathrm{Bi}$ content of $4.7 \%$ in the GaNAsBi epilayer lattice matched with the GaAs substrate was achieved by Wei et al [8] in 2005. The reduction of band gap is well explained using a double BAC model by Lin et al [9]. This paper is organized as follows: section 2 provides the theoretical model and physical parameters for calculation of the electronic band structure, optical properties and the corresponding band parameters. Results 
Table 1. Material parameters.

\begin{tabular}{lcccccccc}
\hline$E_{\mathrm{g}}^{\mathrm{GaAs}}[1]$ & $E_{\mathrm{g}}^{\mathrm{GaN}}[1]$ & $E_{\mathrm{g}}^{\mathrm{GaBi}}[1]$ & $C_{\mathrm{GaAsN}}[1]$ & $C_{\mathrm{GaAsBi}}[1]$ & $C_{\mathrm{GaAsNBi}}[1]$ & $a_{\mathrm{GaAs}}[17]$ & $a_{\mathrm{GaN}}[17]$ & $a_{\mathrm{GaBi}}[18]$ \\
\hline $1.43 \mathrm{eV}$ & $3.26 \mathrm{eV}$ & $-1.45 \mathrm{eV}$ & $20 \mathrm{eV}$ & $6.1 \mathrm{eV}$ & $16.2 \mathrm{eV}$ & $5.653 \AA$ & $4.50 \AA$ & $6.324 \AA$ \\
\hline
\end{tabular}

of 16-band $k p$ model for the bulk alloy GaAsNBi/GaAs are expressed in section 3 . The effects of strain on the band structure have also been discussed in this section and we have also discussed the deviation in band gap energy as a function of $\mathrm{Bi}$ concentration in this section. Section 4 contains the essence of our work contribution related to the relevant area.

\section{Mathematical modelling}

16-band $\mathrm{kp}$ Hamiltonian is used to calculate the band structure of bulk $\mathrm{GaAs}_{1-x-y} \mathrm{~N}_{x} \mathrm{Bi}_{y}$ alloy. Hamiltonian is a combination of valence BAC (VBAC) and conduction BAC (CBAC) [10]. Material parameters used for calculation are provided in table 1. Here, we used 8- and 16-band $k p$ Hamiltonian required for the calculation of the band structure of GaAsNBi/GaAs quantum wells (QWs). This section is organized as follows. In subsection 2.1, theoretical models and material parameters used to determine electronic band structure with different $\mathrm{Bi}$ mole fractions are introduced. The variations of energy and band structure under strain are presented in subsection 2.2. Then, we have calculated the band offset with and without strain in subsection 2.3. In subsection 2.4, the optical gain of $\mathrm{GaAs}_{1-x-y} \mathrm{~N}_{x} \mathrm{Bi}_{y} / \mathrm{GaAs}$ is presented and unstrained energy for $\mathrm{GaAsNBi} / \mathrm{GaAs} \mathrm{QWs}$ [11] is derived from equation (1):

$$
\begin{aligned}
E^{\text {unstrained }}(x, y)= & (1-x-y) \cdot E_{\mathrm{g}}^{\mathrm{GaAs}}+x \cdot E_{\mathrm{g}}^{\mathrm{GaN}} \\
& +y \cdot E_{\mathrm{g}}^{\mathrm{GaBi}}-C_{\mathrm{GaAsN}} x(1-x) \\
& -C_{\mathrm{GaAsBi}} y(1-x)-C_{\mathrm{GaAsNBi}} x y,
\end{aligned}
$$

where $C$ is a bowing coefficient.

\subsection{VBAC and CBAC models}

$\mathrm{Bi}$ and $\mathrm{N}$ incorporation into GaAs perturbed the valence and conduction bands as $\mathrm{Bi}$ generates a resonant impurity state in the valence band as a strongly perturbed host state, while $\mathrm{N}$ produces a resonant impurity state additional to the host states in the conduction band. We are taking into account the effect of VBAC and CBAC perturbations using a 16-band Hamiltonian, by the inclusion of $6 \mathrm{Bi}$ and $2 \mathrm{~N}$ related impurity states to the 8-band Hamiltonian of the host semiconductor matrix [10-15]. This approach has been used to study the $\mathrm{BAC}$ interaction of $\mathrm{Bi}$ and $\mathrm{N}$ impurity model predicting the conduction band minima, as there is a N-related impurity state because of quantum anticrossing interaction due to the upward shift of the heavy hole $(\mathrm{HH}) /$ light hole $(\mathrm{LH})$ and $\mathrm{SO}$ split off due to modification in the valence band structure and
SO-related level bands is well-explained by the VBAC model [13]. The 16-band Hamiltonian reduces to a $6 \times 6$ Hamiltonian at the $\Gamma$ point $(k=0)$ which is as given below [10]:

$$
H_{6 \times 6}=\left[\begin{array}{cccccc}
C^{\prime} & 0 & 0 & V_{\mathrm{N}}(x) & 0 & 0 \\
0 & H^{\prime} / L^{\prime} & 0 & 0 & V_{\mathrm{Bi}}(y) & 0 \\
0 & 0 & S & 0 & 0 & V_{\mathrm{Bi}}(y) \\
V_{\mathrm{N}}(x) & 0 & 0 & E_{\mathrm{N}} & 0 & 0 \\
0 & V_{\mathrm{Bi}}(x) & 0 & 0 & E_{\mathrm{Bi}} & 0 \\
0 & 0 & V_{\mathrm{Bi}}(x) & 0 & 0 & E_{\mathrm{Bi}-\mathrm{SO}}
\end{array}\right],
$$

where $C^{\prime}=E_{\mathrm{g}}^{\mathrm{GaAs}}+\Delta E_{\mathrm{CBM}}(x, y), H^{\prime} / L^{\prime}=\Delta E_{\mathrm{VBM}}(x, y)$; and $S=(1 / 2)\left(H^{\prime}+L^{\prime}\right)-\Delta_{0}^{\mathrm{GaAs}}=\Delta E_{\mathrm{SO}}(x, y), E_{\mathrm{g}}^{\mathrm{GaAs}}$ is unstrained band gap of GaAs and $\Delta E_{\mathrm{CBM}}(x, y), \Delta E_{\mathrm{VBM}}(x, y)$ and $\Delta E_{\mathrm{SO}}(x, y)$ are conduction band offset $(\mathrm{CBO})$, valence band offset (VBO) and SO split off band between the end-point ternary compounds GaAsN and GaAsBi with the following equations:

$$
\begin{aligned}
& \Delta E_{\mathrm{CBM}}(x, y)=x \Delta E_{\mathrm{CBM}}^{\mathrm{GaAsN}}(x, y)+y \Delta E_{\mathrm{CBM}}^{\mathrm{GaAsBi}}(x, y), \\
& \Delta E_{\mathrm{VBM}}(x, y)=x \Delta E_{\mathrm{VBM}}^{\mathrm{GaAsN}}(x, y)+y \Delta E_{\mathrm{VBM}}^{\mathrm{GaAsBi}}(x, y) .
\end{aligned}
$$

In the same way, we can define $\Delta E_{\mathrm{SO}}(x, y)$, the coupling parameters take care $V_{\mathrm{Bi}}(y)=C_{\mathrm{Bi}} \sqrt{y}$ and $V_{\mathrm{N}}(x)=C_{\mathrm{N}} \sqrt{x}$ of the coupling between the $\mathrm{Bi}$ impurity states and the valence band extended states with the host conduction band matrix of the $\mathrm{N}$ resonant states. $E_{\mathrm{N}}$ and $E_{\mathrm{Bi}}$ give the energy of the Nand Bi-related resonant states and $E_{\mathrm{Bi}-\mathrm{SO}}$ is the atomic SO splitting energy for Bi. 6-band Hamiltonian of equation (2) yields six different eigen states corresponding to the splitting of the $\mathrm{CB}, \mathrm{HH} / \mathrm{LH}$ and $\mathrm{SO}$ split-off bands into $E_{+}$and $E_{-}$ sub-bands given by the following set of equations:

$$
\begin{aligned}
& E_{\mathrm{CB} \pm}=\frac{1}{2}\left(C^{\prime}+E_{\mathrm{N}}\right. \\
& \left. \pm \sqrt{\left(C^{\prime 2}-2 C^{\prime} E_{\mathrm{N}}+E_{\mathrm{N}}^{2}+4 V_{\mathrm{N}}(x)^{2}\right)}\right) \\
& E_{\mathrm{HH} / \mathrm{LH} \pm}=\frac{1}{2}\left(H^{\prime}+E_{\mathrm{Bi}}\right. \\
& \left. \pm \sqrt{\left(H^{\prime 2}-2 H^{\prime} E_{\mathrm{Bi}}+E_{\mathrm{Bi}}^{2}+4 V_{\mathrm{Bi}}(y)^{2}\right)}\right) \\
& E_{\mathrm{SO} \pm}=\frac{1}{2}\left(S^{\prime}+E_{\mathrm{BiNSO}}\right. \\
& \left. \pm \sqrt{\left(S^{\prime 2}-2 S^{\prime} E_{\mathrm{BiNSO}}+E_{\mathrm{BiNSO}}^{2}+4 V_{\mathrm{Bi}}(y)^{2}\right)}\right) .
\end{aligned}
$$


The band gap energy of the quaternary alloy $\mathrm{GaAs}_{1-x-y} \mathrm{~N}_{x} \mathrm{Bi}_{y}$ that is a combination of VBAC and CBAC models is given as

$$
\begin{aligned}
& E_{\mathrm{g}}(x, y)=\frac{1}{2}\left[E_{\mathrm{g}}^{\mathrm{GaAs}}+\Delta E_{\mathrm{CBM}}(x, y)\right. \\
& \left.+E_{\mathrm{N}}-\sqrt{\left(C^{\prime}-E_{\mathrm{N}}\right)^{2}+4 C_{\mathrm{N}}^{2} y}\right] \\
& -\frac{1}{2}\left[\Delta E_{\mathrm{VBM}}(x, y)+E_{\mathrm{Bi}}-\sqrt{\left(H^{\prime}-E_{\mathrm{Bi}}^{2}\right)+4 C_{\mathrm{Bi}}^{2} x}\right] .
\end{aligned}
$$

Using Vegard's law, the lattice constant of $\mathrm{GaAs}_{1-x-y} \mathrm{~N}_{x} \mathrm{Bi}_{y}$ can be obtained by linear interpolation of endpoint compound of lattice constant $[16,17]$ :

$$
a_{\mathrm{GaAs}_{1-x-y}} \mathrm{~N}_{x} \mathrm{Bi}_{y}=(1-x-y) a_{\mathrm{GaAs}}+y a_{\mathrm{GaBi}}+x a_{\mathrm{GaN}},
$$

the value of lattice constant is given in table 1 . The band parameters required for the calculation of $\mathrm{VBAC}$ and $\mathrm{CBAC}$ band gap and SO splitting energy for the GaAsNBi are listed in table 2 .

As $k p$ models have some advantages compared with other models like DFT, etc. and also have some limitation: firstly, we are only able to see the energy variation with respect to wave vector in the first Brillouin zone (BZ). Secondly, only certain values of doping atom concentration are feasible in this model if large concentration of doping atom shifts to another model like DFT. In our model, we used 16-band $k p$ Hamiltonian which is the mixture of impurity and potential matrix, and these matrices depend upon end-point ternary compound and coupling parameter, which in turn depends upon the incorporation of Bi concentration into GaAs. Substituting a small percentage of $\mathrm{As}$ by $\mathrm{Bi}$ in $\mathrm{GaAs}$ produces a huge reduction of band gap energy from 60 to $90 \mathrm{meV} / \mathrm{Bi} \%$ of incorporation described by the VBAC model. The band gap reduction in bismide alloys is mainly due to the movement of $\mathrm{HH}$ and $\mathrm{LH}$ bands. However, the position of the split off SO band is found unchanged significantly, resulting in a huge increase in the SO splitting energy $\left(\Delta_{\mathrm{o}}\right)$. This makes $\mathrm{GaAs}_{1-x} \mathrm{Bi}_{x}$ a unique material for possible applications in spintronics. Following this model, incorporation of $\mathrm{Bi}$ in $\mathrm{GaAsBi}$ alloys grown over $\mathrm{GaAs}$ substrates leads to perturbation in the valence band which then leads to the formation of six sub-valence bands which can be grouped in levels $E_{+,(\mathrm{HH}, \mathrm{LH}, \mathrm{SO})}$ and $E_{-,(\mathrm{HH}, \mathrm{LH}, \mathrm{SO})}$. The energy level related to the six above-mentioned sub-bands depends on the Bi Concentration. At $x>10 \% \mathrm{Bi}$, the energy splitting of the split off band exceeds the band gap $\left(\Delta_{\mathrm{o}} \geq E_{\mathrm{g}}\right)$. This feature in the band structure could suppress an important Auger recombination channel and improve the performance of semiconductor lasers having Bi-containing active layers $[5,11,12,22]$.

\subsection{6-band kp model}

Substitution of arsenic atoms in GaAs by $\mathrm{Bi}$ and $\mathrm{N}$ leads to the formation of localized defect states near the valence sub-bands $\mathrm{HH} / \mathrm{LH}, \mathrm{SO}$ and $\mathrm{CB}$ of the host semiconductor in which the energy levels $E_{\mathrm{Bi}}, E_{\mathrm{Bi}-\mathrm{SO}}$ and $E_{\mathrm{N}}$, respectively, were included in the 16-band Hamiltonian. The electronic band structure of $\mathrm{GaAs}_{1-x-y} \mathrm{~N}_{x} \mathrm{Bi}_{y}$ can be determined using this Hamiltonian which is the modified form of the 8-band $k p$ Hamiltonian based on the eight band second-order Kane model applied to the zinc blended structure and Löwdin perturbation theory [16]. The resultant $(16 \times 16)$ matrix of the Hamiltonian can be written as $[9,14]$ :

$$
H_{16 \times 16}=\left[\begin{array}{cc}
H_{8 \times 8}^{\mathrm{mod}} & V_{8 \times 8}^{\mathrm{N}, \mathrm{Bi}}(y, x) \\
V_{8 \times 8}^{\mathrm{N}, \mathrm{Bi}}(y, x) & E_{8 \times 8}^{\mathrm{mod}}
\end{array}\right],
$$

where $H_{8 \times 8}$ is modified with strain effect include $8 \times 8$ matrix given below:

$$
H_{8 \times 8}=\left[\begin{array}{cccccccc}
C & 0 & -\frac{1}{\sqrt{2}} P_{+} & \sqrt{\frac{2}{3} P_{Z}} & \frac{1}{\sqrt{6}} P_{-} & 0 & \frac{1}{\sqrt{3}} P_{Z} & \frac{1}{\sqrt{3}} P_{-} \\
0 & C & 0 & \frac{1}{\sqrt{6}} P_{+} & \sqrt{\frac{2}{3} P_{Z}} & \frac{1}{\sqrt{2}} P_{-} & \frac{1}{\sqrt{3}} P_{+} & -\frac{1}{\sqrt{3}} P_{Z} \\
-\frac{1}{\sqrt{2}} P_{+}^{*} & 0 & H & \alpha & \beta & 0 & \frac{i \alpha}{\sqrt{2}} & -i \sqrt{2} \beta \\
\sqrt{\frac{2}{3} P_{Z}} & \frac{1}{\sqrt{6}} P_{+}^{*} & \alpha^{*} & L & 0 & \beta & i\left(\sqrt{2} \varepsilon-\frac{D}{\sqrt{2}}\right) & i \alpha \sqrt{\frac{3}{2}} \\
\frac{1}{\sqrt{6}} P_{-}^{*} & \sqrt{\frac{2}{3} P_{Z}} & \beta^{*} & 0 & L & -\alpha & -i \alpha^{*} \sqrt{\frac{3}{2}} & i\left(\sqrt{2} \varepsilon-\frac{D}{\sqrt{2}}\right) \\
0 & -\frac{1}{\sqrt{2}} P_{-}^{*} & 0 & \beta^{*} & -\alpha^{*} & H & -i \sqrt{2} \beta^{*} & -\frac{i \alpha^{*}}{\sqrt{2}} \\
\frac{1}{\sqrt{3}} P_{Z} & -\frac{1}{\sqrt{2}} P_{+}^{*} & \frac{i \alpha^{*}}{\sqrt{2}} & i\left(-\sqrt{2} \varepsilon+\frac{D}{\sqrt{2}}\right) & i \alpha \sqrt{\frac{3}{2}} & i \sqrt{2} \beta & S & 0 \\
\frac{1}{\sqrt{3}} P_{-}^{*} & -\frac{1}{\sqrt{3}} P_{Z} & i \sqrt{2} \beta^{*} & -i \alpha \sqrt{\frac{3}{2}} & i\left(\sqrt{2} \varepsilon-\frac{D}{\sqrt{2}}\right) & \frac{i \alpha}{\sqrt{2}} & 0 & S
\end{array}\right],
$$


Table 2. Band parameters of GaAsN and GaAsBi alloys for VBAC and BAC calculations.

\begin{tabular}{lc} 
Parameter & Considered value (eV) \\
\hline$E_{\mathrm{N}}$ & $0.89[22]$ \\
$C_{\mathrm{N}}$ & $2.83[22]$ \\
$E_{\mathrm{Bi}}$ & $-1.25[19]$ \\
$E_{\mathrm{Bi}-\mathrm{SO}}$ & $-2.7[19]$ \\
$C_{\mathrm{Bi}}$ & $1.01[19]$ \\
$E^{\mathrm{GaAs}}$ & $1.43[20]$ \\
${ }^{\mathrm{GaAs}}$ & $0.341[20]$ \\
$E_{\mathrm{P}}^{\mathrm{GaAs}}$ & $28.8[20]$ \\
$\Delta E_{\mathrm{CBM}}^{\mathrm{GaAsN}}$ & $0.07[17]$ \\
$\Delta E_{\mathrm{VBM}}^{\mathrm{GaAsN}}$ & $1.84[17]$ \\
$\Delta E_{\mathrm{SO}}^{\mathrm{GaAsN}}$ & $1.52[17]$ \\
$\Delta E_{\mathrm{CBM}}^{\mathrm{GaAsBi}}$ & $-2.30[21]$ \\
$\Delta E_{\mathrm{VBM}}^{\mathrm{GaAsBi}}$ & $0.6[21]$ \\
$\Delta E_{\mathrm{SO}}^{\mathrm{GaAsBi}}$ & $-1.3[21]$ \\
\hline
\end{tabular}

where

$$
\begin{aligned}
& C=E_{\mathrm{g}}+\frac{\hbar^{2}}{2 m_{0}}\left[\frac{1}{m_{e}^{*}}-\frac{E_{P}}{3}\left(\frac{2}{E_{\mathrm{g}}}-\frac{1}{E_{\mathrm{g}}+\Delta_{\mathrm{SO}}}\right)\right] \\
& \times\left(K_{x}^{2}+K_{y}^{2}+K_{z}^{2}\right)+\Delta E_{\mathrm{CBM}}(x, y)+\varepsilon_{1}(z), \\
& H=\frac{-\hbar^{2}}{2 m_{0}}\left[\left(K_{x}^{2}+K_{y}^{2}\right)\left(\gamma_{1}+\gamma_{2}\right)+K_{z}^{2}\left(\gamma_{1}-2 \gamma_{2}\right)\right] \\
& +\Delta E_{\mathrm{VBM}}(x, y)+\varepsilon_{2}(z), \\
& L=\frac{-\hbar^{2}}{2 m_{0}}\left[\left(K_{x}^{2}+K_{y}^{2}\right)\left(\gamma_{1}-\gamma_{2}\right)+K_{z}^{2}\left(\gamma_{1}+2 \gamma_{2}\right)\right] \\
& +\Delta E_{\mathrm{VBM}}(x, y)-\varepsilon_{2}(z), \\
& S=1 / 2(L+H)-\Delta_{\mathrm{SO}}-\Delta E_{\mathrm{SO}}(x, y)-\varepsilon_{2}(z), \\
& D=L-H \text {, } \\
& P_{ \pm}=P\left(K_{x} \pm i K_{y}\right)-P\left(e_{x x} K_{x} \pm i e_{y y} K_{y}\right) \text {, } \\
& P_{z}=P K_{z}-P\left(e_{z z} K_{z}\right), \\
& \alpha=\frac{\hbar^{2}}{2 m_{0}} 2 \sqrt{3}\left[K_{z}\left(K_{x}-i K_{y}\right) \gamma_{3}\right], \\
& \beta=\frac{\hbar^{2}}{2 m_{0}} \sqrt{3}\left[\left(K_{x}^{2}+K_{y}^{2}\right) \gamma_{2}-2 i K_{x} K_{y} \gamma_{3}\right], \\
& \varepsilon_{1}(z)= \begin{cases}2\left(a_{\mathrm{c}}+a_{\mathrm{v}}\right)\left(1-C_{12} / C_{11}\right) e_{x x} & \text { in the well, } \\
0 & \text { in the barrier, }\end{cases} \\
& \varepsilon_{2}(z)= \begin{cases}b\left(1+2 C_{12} / C_{11}\right) e_{x x} & \text { in the well, } \\
0 & \text { in the barrier. }\end{cases}
\end{aligned}
$$

Here $C, H, L$ and $S$ are modified with band offsets and related to strain parameters $\Delta E(x, y)$ and $\varepsilon(z)$, respectively. In equation (10), $a_{\mathrm{c}}$ and $a_{\mathrm{v}}$ are the hydrostatic deformation potential for conduction and valence band, respectively, $b$ is the shear deformation potential and $C_{11}$ and $C_{12}$ are the elastic stiffness constants:

$$
e_{x x}=\frac{a^{\prime}-a M}{a M},
$$

$e_{x x}$ is in plane strain where $a^{\prime}$ and $a M$ are the lattice constants for the substrate and well layer, respectively. $P$ is the Kane matrix element and is normally expressed in terms of electron volt (eV) as below [19-22]:

$$
E_{\mathrm{p}}=\frac{2 m_{0}}{\hbar^{2}} P^{2}
$$

In the $8 \times 8$ Hamiltonian, valence band parameters related to the Luttinger parameters are used in the $6 \times 6$ valence band Hamiltonian due to inclusion of the conduction band states in the $8 \times 8$ Hamiltonian [22]. The relation between the modified Luttinger parameters and the Luttinger parameters related to VBAC is given below [23,24]:

$$
\begin{aligned}
& \gamma_{1}=\gamma_{1}^{\mathrm{VB}}-\frac{E_{\mathrm{p}}}{3 E_{\mathrm{g}}}, \\
& \gamma_{2}=\gamma_{2}^{\mathrm{VB}}-\frac{E_{\mathrm{p}}}{6 E_{\mathrm{g}}}, \\
& \gamma_{3}=\gamma_{3}^{\mathrm{VB}}-\frac{E_{\mathrm{p}}}{6 E_{\mathrm{g}}} .
\end{aligned}
$$

\subsection{Strain-related $V B$ and $C B$ shifts for GaAsNBi/GaAs $Q W s$}

The conduction band and valence band edges shifted due to the effect of hydrostatic and shear strain in GaAsNBi/GaAs QWs. Strain-induced ones shift the conduction band minimum and the valence band maxima calculated using the Bir-Pikus Hamiltonian [25], and are responsible for the quantum confinement of electrons and holes in QW structures. The shear component of strain that causes the separation between the valence sub-bands $E_{\mathrm{VB}-\mathrm{HH}}$ and $E_{\mathrm{VB}-\mathrm{LH}}$ by removing the valence band degeneracy is given as [26]:

$$
\begin{aligned}
E_{\mathrm{CB}}(\varepsilon) & =E_{\mathrm{CB}}(\varepsilon=0)+\Delta E_{\mathrm{CB}}^{\mathrm{hy}}, \\
E_{\mathrm{VB}-\mathrm{HH}}(\varepsilon) & =E_{\mathrm{VB}}(\varepsilon=0)+\Delta E_{\mathrm{VB}}^{\mathrm{hy}}-1 / 2 \Delta E_{\mathrm{s}}, \\
E_{\mathrm{VB}-\mathrm{LH}}(\varepsilon) & =E_{\mathrm{VB}}(\varepsilon=0)+\Delta E_{\mathrm{VB}}^{\mathrm{hy}}+1 / 2 \Delta E_{\mathrm{s}},
\end{aligned}
$$

where $\Delta E_{\mathrm{CB}}^{\mathrm{hy}}=2 a_{\mathrm{c}}\left(1-\frac{c_{12}}{c_{11}}\right) e_{x x}$ and $\Delta E_{\mathrm{VB}}^{\mathrm{hy}}=2 a_{\mathrm{v}}$ $\times\left(1-\frac{c_{12}}{c_{11}}\right) e_{x x}$ influence of hydrostatic components of strain on the conduction and valence bands, respectively and $\Delta E_{\mathrm{s}}=$ $2 b\left(1+\frac{c_{12}}{c_{11}}\right) e_{x x}$ contribute shearing of strain to the band structure. 

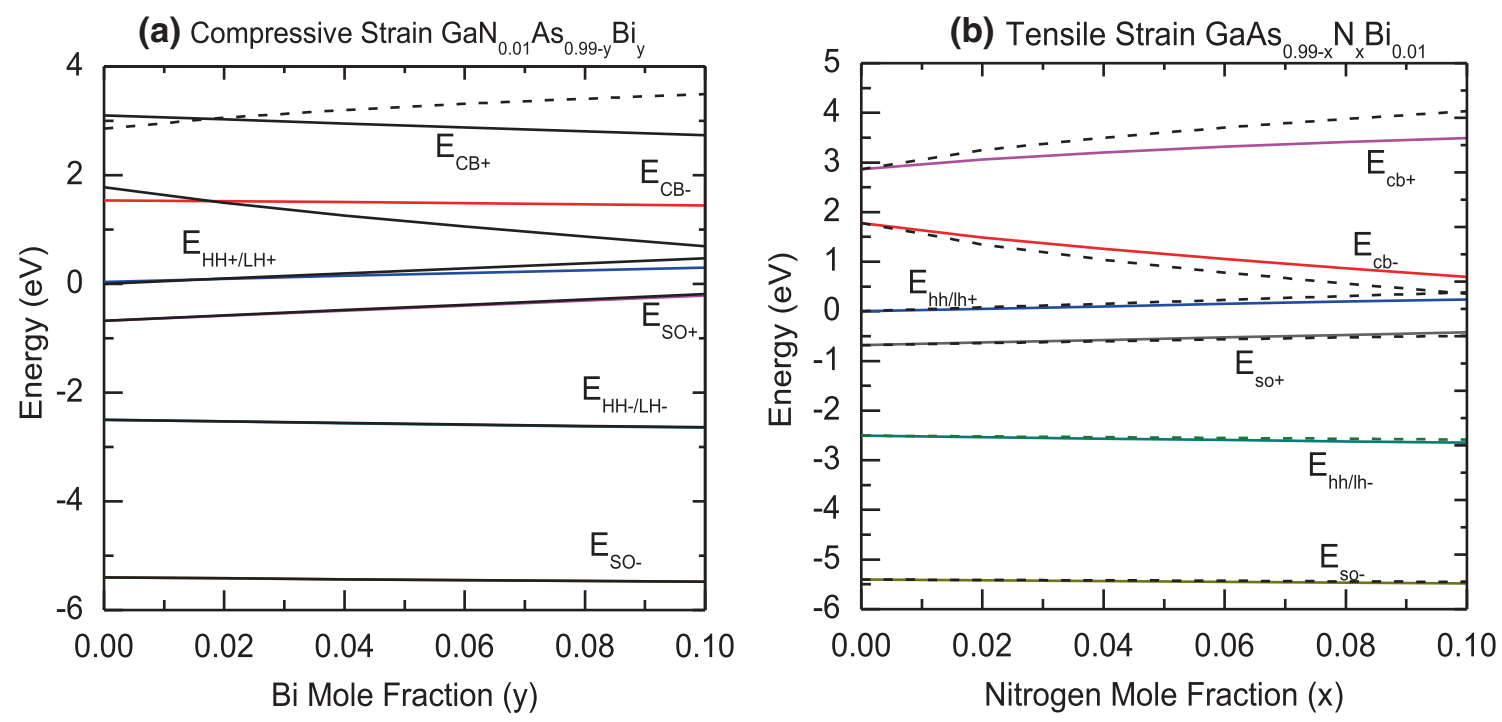

Figure 1. Energy level positions of $E \pm$ with respect to $\mathrm{CB}, \mathrm{HH}, \mathrm{LH}$ and $\mathrm{SO}$ bands as a function of $\mathrm{Bi}$ and $\mathrm{N}$ mole fractions for (a) compressive-strained and (b) tensile-strained GaAsNBi/GaAs QWs at room temperature $(T=300 \mathrm{~K})$. Dashed lines represent the lattice-matched conditions.

\subsection{Optical gain}

Optical gain $g(\hbar \omega)$ for GaAsNBi/GaAs QWs is calculated by taking into account all the transitions between respective sub-bands as per density matrix theory $[27,28]$. A conformist method [29] based on the intraband relaxation time $\left(\tau_{\text {in }}\right)$ approximation convoluted with a Lorentzian function with suitable broadening time $\left(1 \times 10^{-14} \mathrm{~s}\right)$ is used to evaluate optical gain spectra [30] given as:

$$
\begin{aligned}
g(\hbar \omega)= & \frac{\pi q^{2}}{n_{\mathrm{r}} c \omega L_{\mathrm{w}} \epsilon_{0} m_{0}^{2}} \sum_{n, m} \int_{0}^{\infty} \frac{k_{\mathrm{p}} d k_{\mathrm{p}}}{2 \pi}\left|M_{n m}\left(k_{\mathrm{p}}\right)\right|^{2} \\
& \times L\left(E_{n, m}^{\mathrm{c}, \mathrm{v}}\left(k_{\mathrm{p}}\right)\right)\left\{f_{n}^{\mathrm{c}}\left(E_{n}^{\mathrm{c}}\left(k_{\mathrm{p}}\right)\right)-f_{m}^{\mathrm{v}}\left(E_{m}^{\mathrm{v}}\left(k_{\mathrm{p}}\right)\right)\right\},
\end{aligned}
$$

where $c$ and $\epsilon_{0}$ are the velocity of light and relative permittivity in free space, $n_{\mathrm{r}}$ is the refractive index of the QW, $L_{\mathrm{w}}$ is the thickness of the $\mathrm{QW}, k_{\mathrm{p}}$ is the in-plane wave vector, $M_{n m}\left(k_{\mathrm{p}}\right)$ is the momentum matrix element between the $m$ th valence sub-band and $n$th conduction band sub-band. $f_{n}^{\mathrm{c}}$ and $f_{m}^{v}$ are the Fermi-Dirac distribution for electrons and holes, given as

$$
\begin{aligned}
& f_{n}^{\mathrm{c}}\left(k_{\mathrm{p}}\right)=\left[1+\exp \left\{\frac{E_{n}^{\mathrm{c}}\left(k_{\mathrm{p}}\right)-E_{\mathrm{f}}^{\mathrm{c}}}{k_{\mathrm{B}} T}\right\}\right]^{-1}, \\
& f_{m}^{\mathrm{v}}\left(k_{\mathrm{p}}\right)=\left[1+\exp \left\{\frac{E_{m}^{\mathrm{v}}\left(k_{\mathrm{p}}\right)-E_{\mathrm{f}}^{\mathrm{v}}}{k_{\mathrm{B}} T}\right\}\right]^{-1} .
\end{aligned}
$$

The Lorentzian function defined in [26] is as follows:

$$
L\left(E_{n, m}^{\mathrm{c}, \mathrm{v}}\left(k_{\mathrm{p}}\right)\right)=\frac{1}{\pi} \frac{\hbar / \tau_{\text {in }}}{\left[E_{n}^{\mathrm{c}}\left(k_{\mathrm{p}}\right)-E_{m}^{\mathrm{v}}\left(k_{\mathrm{p}}\right)-\hbar \omega\right]^{2}+\left(\frac{\hbar}{\tau_{\mathrm{in}}}\right)^{2}} .
$$

where $E_{n}^{\mathrm{c}}$ and $E_{m}^{\mathrm{v}}$ are the energy dispersion curves of the $n$th and the $m$ th sub-bands, respectively, consider the barrier effecting [30-32], $E_{\mathrm{f}}^{\mathrm{c}}$ and $E_{\mathrm{f}}^{\mathrm{v}}$ are the quasi-Fermi levels of corresponding bands.

\section{Results and discussion}

Figure 1 shows the variation of $E_{+}$and $E_{-}$energy levels related to the $\mathrm{CB}$ and $\mathrm{VB}$ in strained $\mathrm{GaAsNBi} / \mathrm{GaAs} \mathrm{QWs}$. Figure 1a shows the variation of energy level which is linear in compressive strain and figure $1 \mathrm{~b}$ shows the deviation under tensile strained with respect to the dashed line under lattice matched conditions is more considerable, showing the effect of $\mathrm{N}$ on band structure is more than Bi impurity and non-linear variation of $E_{\mathrm{CB}+}$ and $E_{\mathrm{CB}-}$ in tensile-strained. For unstrained conditions in figure 1 a (lattice-matched conditions as shown by the dashed line), addition of $\mathrm{Bi}$ and $\mathrm{N}$ into GaAs, pushes the energy level $E_{\mathrm{CB}-}$ in the downward direction nearly about $100 \mathrm{meV} / \% \mathrm{Bi}$ and $E_{\mathrm{HH}} / L_{\mathrm{H}+}$ in the upward direction by $47 \mathrm{meV} / \% \mathrm{Bi}$. The SO split off energy level $E_{\mathrm{SO}+}$ moves upward by nearly $6 \mathrm{meV} / \% \mathrm{Bi}$ which shows that there is a slight increase of the SO splitting energy.

Figure 2 presents the difference in the band structure of tensile- and compressive-strained GaAsNBi/GaAs QW structures. These strains of band structure are plotted for $\mathrm{N}$ and $\mathrm{Bi}$ concentration ratios of 5 and 0.2 , respectively, which is greater than or less than the matched lattice $\mathrm{N}$ to $\mathrm{Bi}$ concentration ratio of 0.58 . The band gap is calculated to be lower as $0.47 \mathrm{eV}$ under the tensile-strained $\mathrm{GaAs}_{0.94} \mathrm{~N}_{0.05} \mathrm{Bi}_{0.01} / \mathrm{GaAs}$ QWs; however, in the case of compressive-strained $\mathrm{GaAs}_{0.94} \mathrm{~N}_{0.01} \mathrm{Bi}_{0.05} / \mathrm{GaAs}$ QWs where such considerable splitting is not observed and band gap with a 


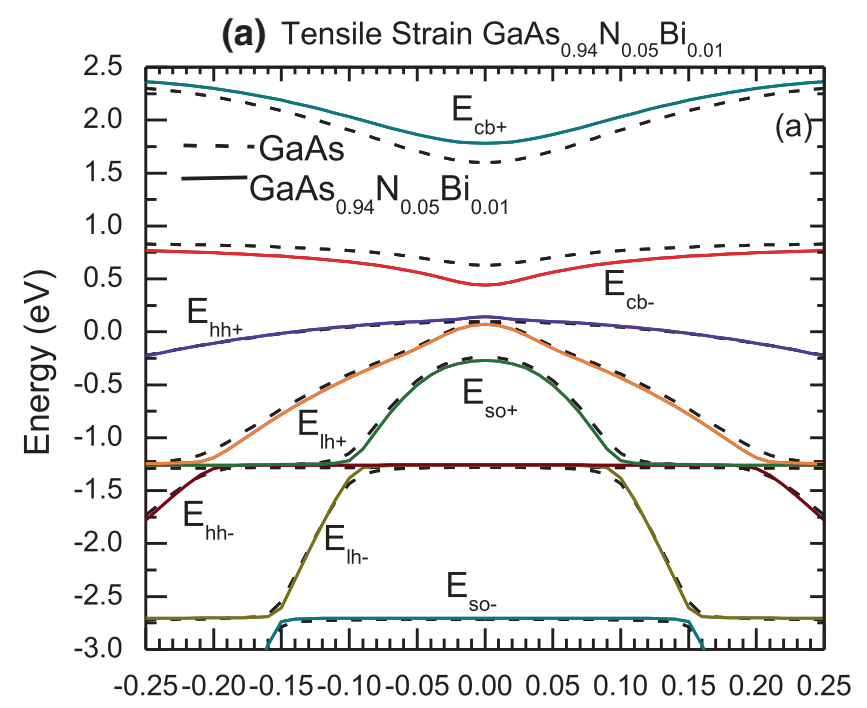

Wave vector $(\mathrm{nm})^{-1}$

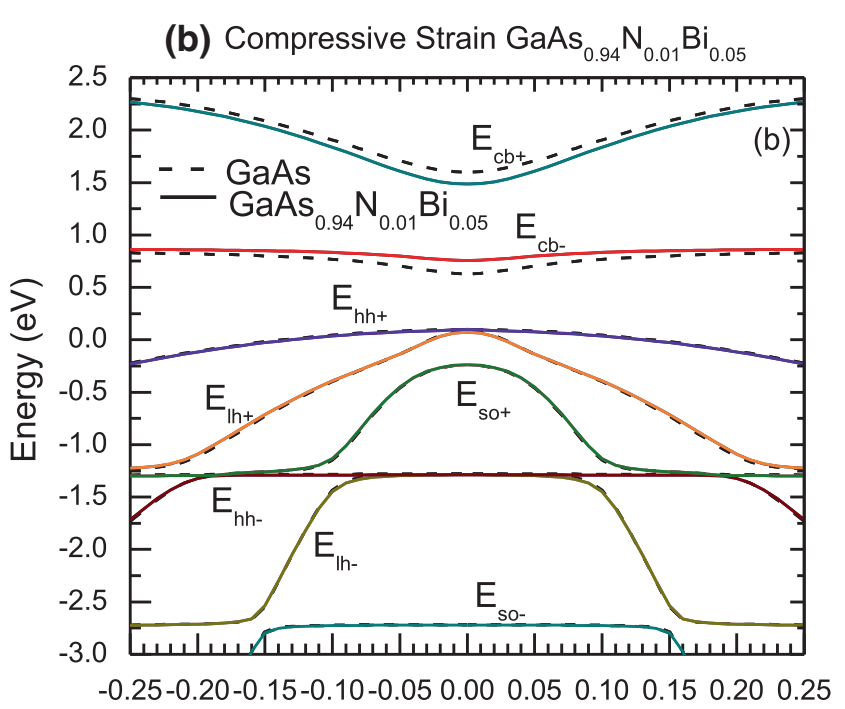

Wave vector $(\mathrm{nm})^{-1}$

Figure 2. Band structure of (a) tensile-strained and (b) compressive-strained GaAsNBi/GaAs QWs in the $k$ direction near the $\Gamma$ point at $T=300 \mathrm{~K}$. Dashed lines indicate the band structure of GaAsNBi alloy lattice-matched with the GaAs substrate.

higher value of $0.78 \mathrm{eV}$ is obtained. The separation between $\mathrm{CB} E_{+}$and $E_{-}$energy levels during generation of tensile strain indicates the effect of large concentration of $\mathrm{N}$ atoms on the $\mathrm{CB}$ of $\mathrm{GaAsNBi} / \mathrm{GaAs}$ QWs as shown in figure 2 (lattice-matched conditions shown by the dashed line). The comparison of band gap energy for $\mathrm{GaAs}_{0.931} \mathrm{~N}_{0.029} \mathrm{Bi}_{0.05}$ at $1.3 \mathrm{eV}$ with that of $\mathrm{GaAs}_{0.977} \mathrm{~N}_{0.023}$ at $1.11 \mathrm{eV}$ [33] shows that the introduction of $\mathrm{Bi}$ impurity atoms in GaAs increases the $\mathrm{N}$ content from 2.3 to 2.9 at $\%$. Hence, this nitrogen composition improves the structural and optical properties of GaAsNBi.

Figure $3 \mathrm{a}-\mathrm{d}$ shows the plot of band offset of GaAsNBi under unstrained conditions, here, described $\mathrm{CBO}$ and VBO correspond to their band offset ratio of the valence and conduction bands. From figure $3 a$ and $b$, we observed that CBO $(470 \mathrm{eV})$ is five times of VBO $(220 \mathrm{eV})$ and both increase with increase in $\mathrm{N}$ and $\mathrm{Bi}$ concentrations is useful for optoelectronic designing purpose and provide better for electron confinement. From figure $3 \mathrm{c}$ and d, we observed that, unlike in figure $3 \mathrm{a}$ and $\mathrm{b}$, here the $\mathrm{VBO}$ and $\mathrm{CBO}$ ratio increases nonlinearly. Here, we observed that the $\mathrm{CBO}$ ratio decreases with increase in $\mathrm{Bi}$ and $\mathrm{N}$ concentrations, but VBO increases with concentration.

Using equation (13), the calculated band offset under strain condition shows some important characteristics. The band offset energy difference between BOHH and BOLH is of $\sim 74 \mathrm{meV}$ and increase with Bi concentration, whereas BOCB decreases below $700 \mathrm{meV}$. From figure $4 \mathrm{a}$, we observed that the value of BOCB decrease with increasing Bi mole fraction for $\mathrm{N}=0$ and $1 \%$ but for other $\mathrm{N} \%$ it seems to be constant. Band offsets of BOHH and BOLH decrease with increase in $\mathrm{N}$ and $\mathrm{Bi}$ mole fractions because of the opposite effect of shearing of strain on $\mathrm{HH}$ and $\mathrm{LH}$ bands as shown in figure 4.
Figure 5 displays the variation in band gap as a function of Bi mole fraction at $k=0$ ( $\Gamma$ point) and decreases with increase in Bi concentration. Therefore, we can tune the band gap for different wavelength regions by changing the Bi concentration. A comparison of our results with experimental data and literature results $[11,34,35]$ is shown in figure 5 . From the figure, we observed the deviation of energy with respect to Bi concentration and the deviation mainly has two reasons: firstly, here, we have used different values of coupling parameter for $\mathrm{N}$ and $\mathrm{Bi}$ about $2.83 \mathrm{eV}$ [22] and $1.01 \mathrm{eV}$ [18], respectively, whereas Bousbih et al [25] and Alberi et al [13] reported the values of 2.7 and $1.55 \mathrm{eV}$, respectively. Here, we used the values of $E_{\mathrm{N}}$ and $E_{\mathrm{Bi}}$ for our work about $0.89 \mathrm{eV}$ and $-1.25 \mathrm{eV}$ [19], respectively, while Bousbih et al [25] reported $E_{\mathrm{N}}$ of $1.65 \mathrm{eV}$ and Alberi et al [13] reported $E_{\mathrm{Bi}}$ of $-0.4 \mathrm{eV}$. Secondly, the deviation may be due to different derivation methods used to design the programme for the calculation of energy.

Figure 6 shows the gain plot that is a function of photon energy for different values of carrier concentration. The optical gain is plotted against photon energy as a function of Carrier concentration. We observed four peaks that can be attributed to the interband transitions that occurred between the excited state $\mathrm{HH} / \mathrm{LH}$ and conduction sub-band. $\mathrm{HH}$ to $\mathrm{LH}$ interband transitions are important for absorption of normal incident light by holes in GaAs [33]. The inter sub-band transitions in the conduction band ranges from 0.20 to $0.60 \mathrm{eV}$ whereas the transitions between the $\mathrm{LH} \rightarrow \mathrm{HH} / \mathrm{HH} \rightarrow \mathrm{HH} / \mathrm{LH} \rightarrow \mathrm{LH}$ sub-bands lie in the range of $0.14-0.90 \mathrm{eV}$.

For different values of well width within a fixed value of carrier density, the active region of the QW causes a shift in peak energy corresponding to gain towards the lower 
(a)

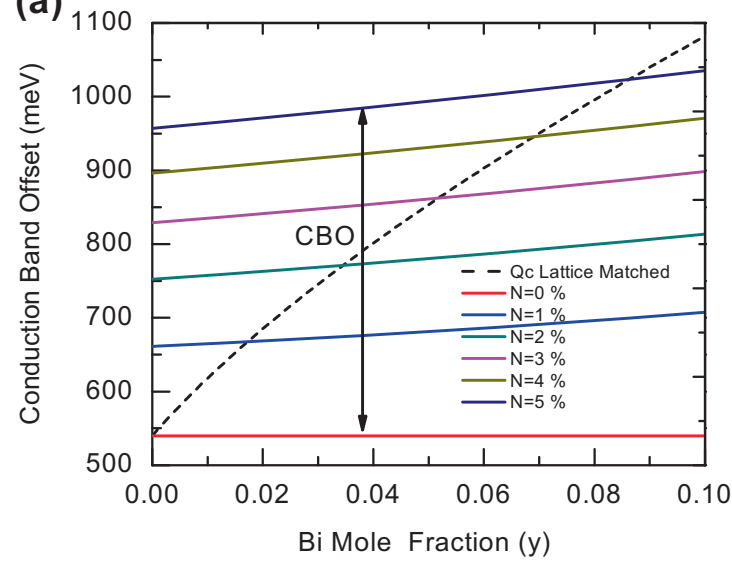

(c)

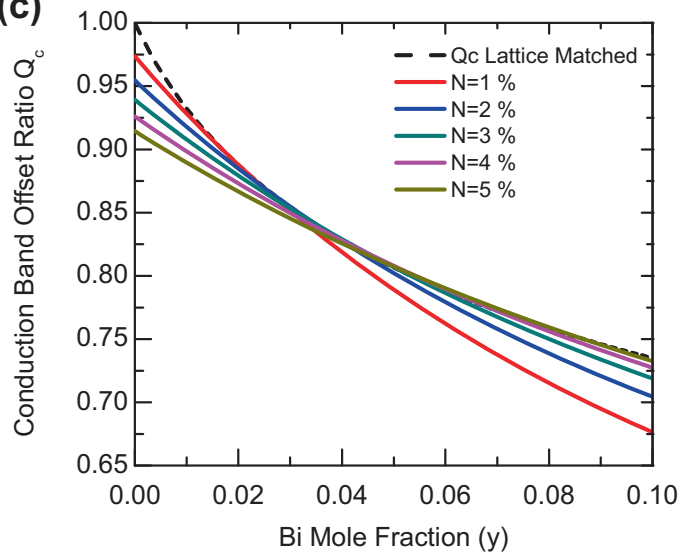

(b)

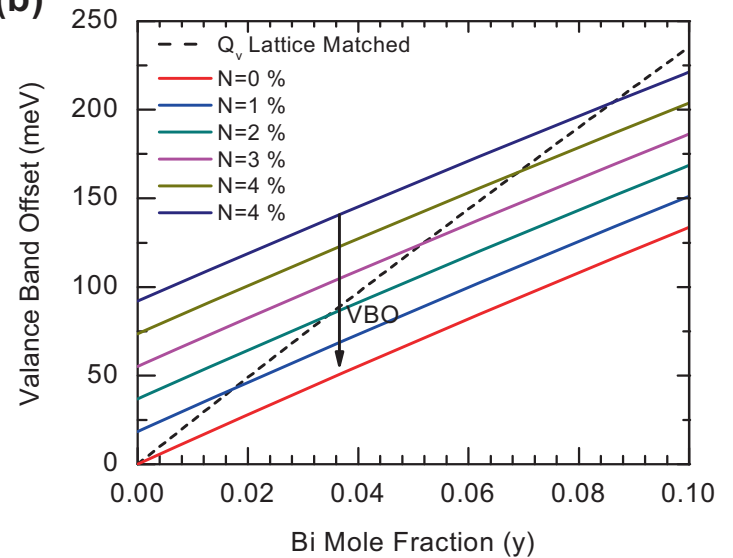

(d)

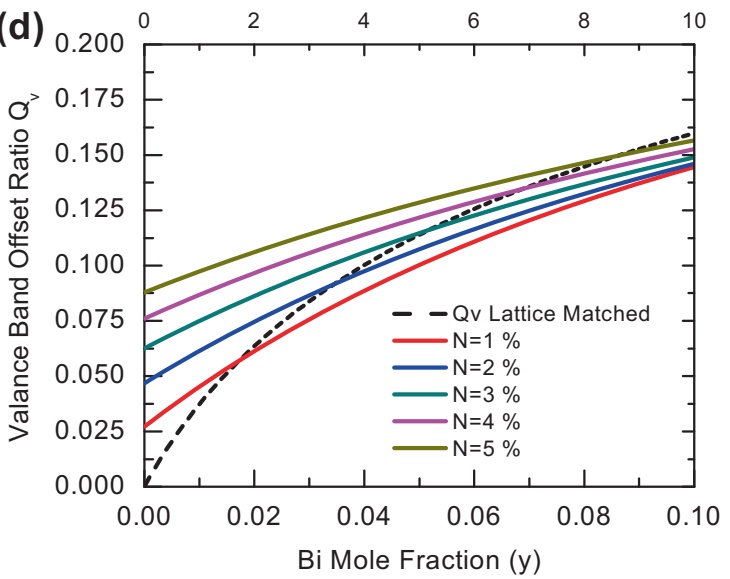

Figure 3. Variation of (a) $\mathrm{CBO}$, (b) $\mathrm{VBO},(\mathbf{c}) \mathrm{CBO}$ ratio and (d) $\mathrm{VBO}$ ratio for GaAsNBi with Bi mole fraction (y) for different values of $\mathrm{N}$ concentration in the material. Dashed lines are used to represent the lattice-matched conditions $(x / y=0.58)$.

(a)

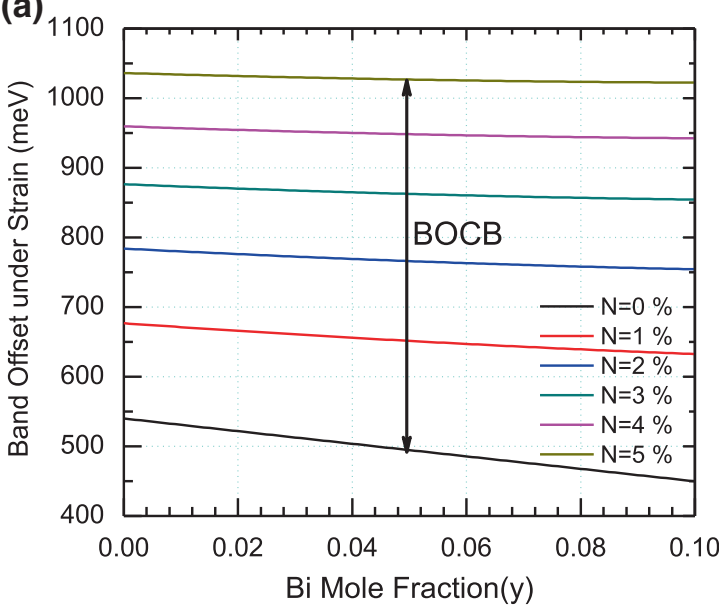

(b)

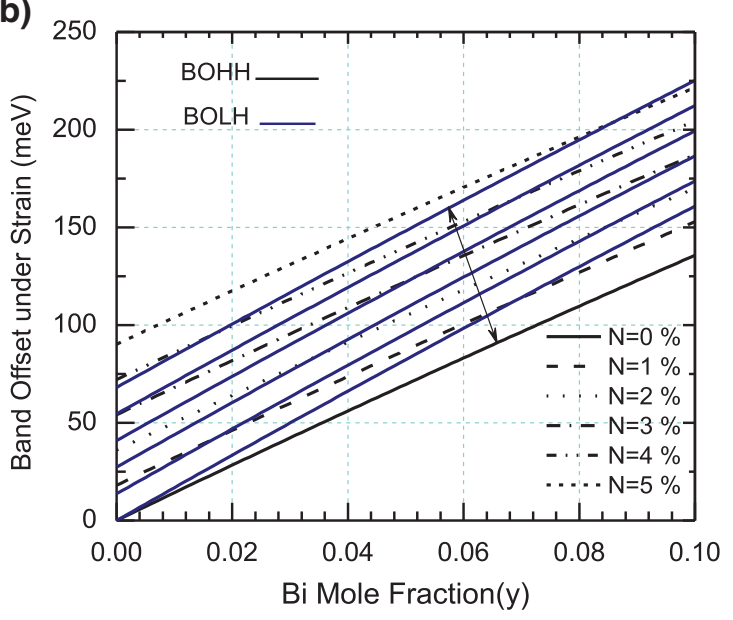

Figure 4. (a) The band offset of conduction band (BOCB), (b) heavy hole (BOHH) and light hole (BOLH) under strain conditions as a function of $\mathrm{Bi}$ mole fraction under different nitrogen concentrations.

wavelength; for fixed value of carrier density, this occurs because energy of sub-band is inversely proportional to the well width. Stronger carrier confinement occurs as the overlap of the exciton wave function increases, and transition energy of the carrier that shift the peak gain towards lower wavelength as shown in figure 7. 


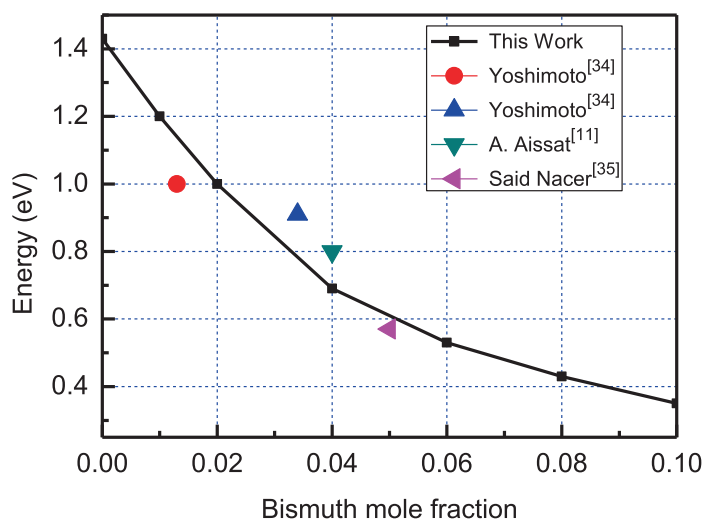

Figure 5. Comparison between calculated and experimental [34] and from the literature $[11,35]$ value of band gap for GaAsNBi as a function of bismuth concentration at $T=300 \mathrm{~K}$.

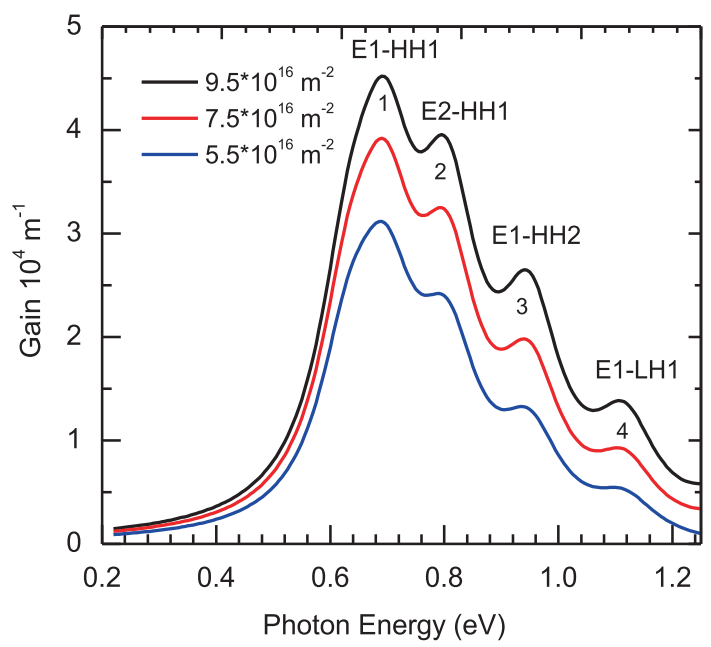

Figure 6. Optical gain plot against photon energy for different values of carrier concentrations exhibiting the interband transitions in $\mathrm{GaAs}_{0.937} \mathrm{~N}_{0.023} \mathrm{Bi}_{0.040} / \mathrm{GaAs} \mathrm{QWs}$ with the barrier effect of GaAs.

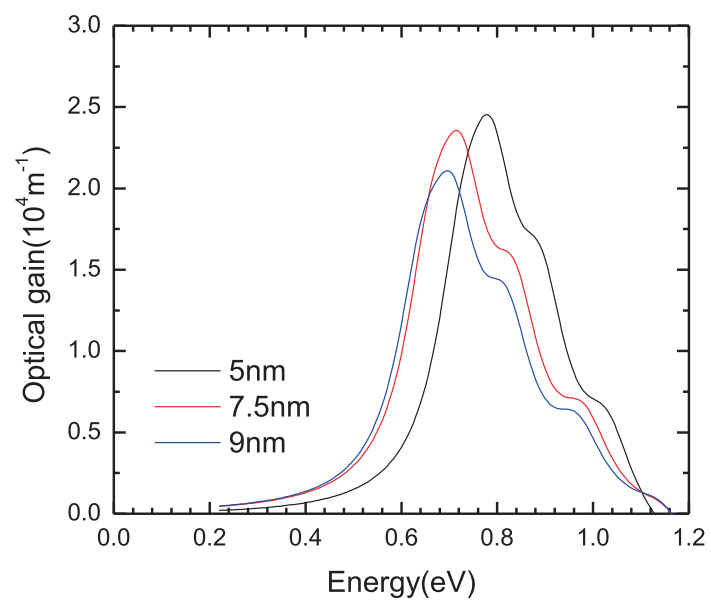

Figure 7. Optical gain considered barrier effect of GaAs in the $\mathrm{GaAs}_{0.937} \mathrm{~N}_{0.023} \mathrm{Bi}_{0.040} / \mathrm{GaAs} \mathrm{QW}$ s for different values of width for the fixed value of carrier concentration of $3 \times 10^{16} \mathrm{~m}^{-2}$.

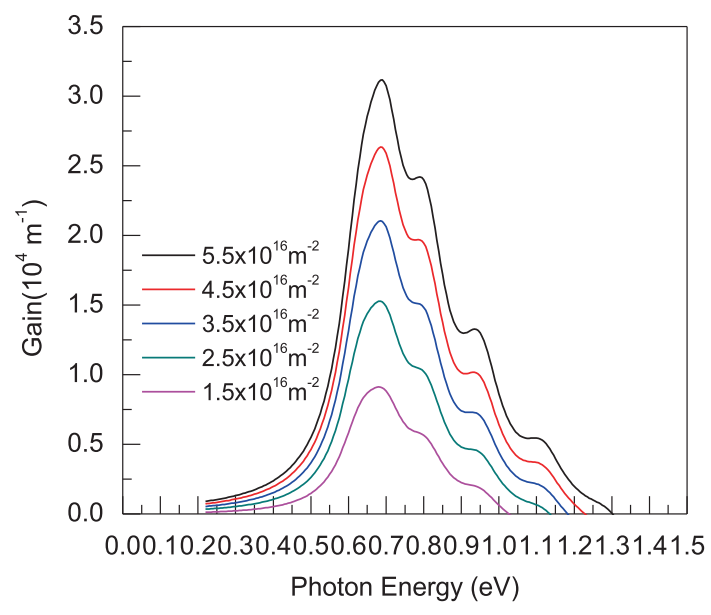

Figure 8. Optical gain spectra having $10 \mathrm{~nm}$ width of the $\mathrm{GaAs}_{0.937} \mathrm{~N}_{0.023} \mathrm{Bi}_{0.040} / \mathrm{GaAs} \mathrm{QWs}$ considered barrier effect of $\mathrm{GaAs}$, for different values of carrier concentrations.

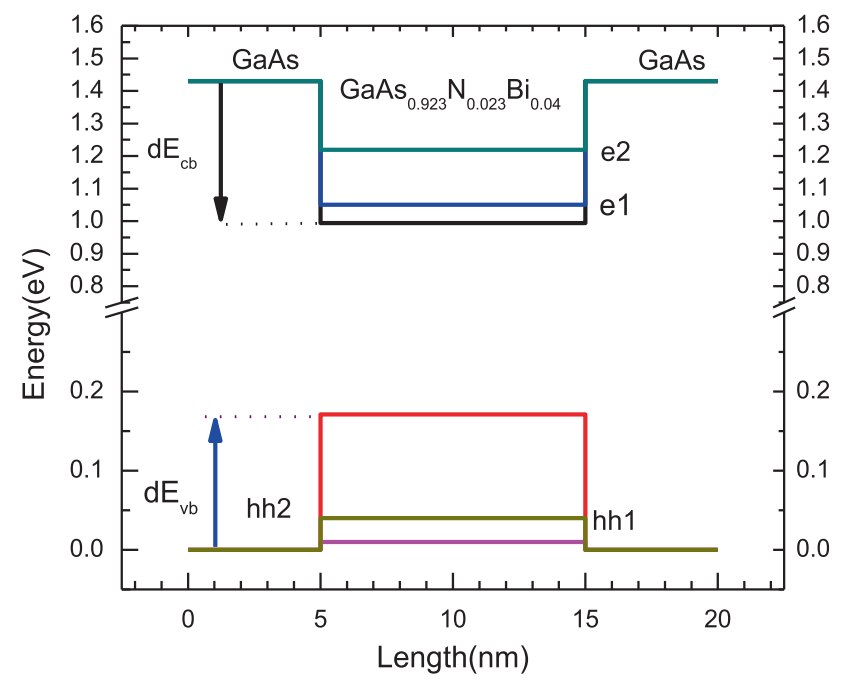

Figure 9. Quantum confinement potential and energy levels for electrons and holes for $10 \mathrm{~nm}$ wide $\mathrm{GaAsNBi} / \mathrm{GaAs}$ QWs under lattice-matched conditions.

Optical gain variation with different values of carrier density of the $\mathrm{GaAs}_{0.937} \mathrm{~N}_{0.023} \mathrm{Bi}_{0.040} / \mathrm{GaAs}$ QWs with a $10 \mathrm{~nm}$ active layer is shown in figure 8. As we see from the graph, increasing the rate of carrier density leads to improvement in the difference of the Fermi function in the mid-infrared region. For optoelectronic devices like sub-band energy, it will act as a tuning parameter. We also observed from the graph that with fixed width of QWs and varying carrier density causes the shift in peak towards lower wavelength.

The quantum confinement potential and the positions of $\mathrm{CB}$ and VB energy levels for lattice matched $10 \mathrm{~nm}$-wide $\mathrm{GaAsNBi} / \mathrm{GaAs} \mathrm{QWs}$ and the dispersion of the corresponding telecommunication window $(1.55 \mu \mathrm{m})$ are shown in figure 9 . 


\section{Conclusions}

Band structure of GaAsNBi alloy lattice matched with GaAs substrates and band structure was calculated using 16-band $k p$ Hamiltonian. VBO and CBO as well their ratios are calculated as a function of both $\mathrm{Bi}$ and $\mathrm{N}$ mole fractions. $\mathrm{CBO}$ as high as $470 \mathrm{meV}$ and VBO of $220 \mathrm{meV}$ are obtained which can improve the device characteristics by providing strong carrier confinement. Band gap values of 0.47 and $0.78 \mathrm{eV}$ are obtained for tensile-strained $\mathrm{GaAs}_{0.94} \mathrm{~N}_{0.05} \mathrm{Bi}_{0.01} / \mathrm{GaAs} \mathrm{QWs}$ and compressive-strained $\mathrm{GaAs}_{0.94} \mathrm{~N}_{0.01} \mathrm{Bi}_{0.05} / \mathrm{GaAs} \mathrm{QWs}$, respectively. Injected charge carrier density increases with an increase in the optical gain of QWs, but for constant value of injected charge carrier density with increase in the dimension of QWs, optical gain decreases because of reduction in the density of state in the sub-band. Hence, the reduction in width of quantum well and peak of optical gain shows the blueshift. Bi and $\mathrm{N}$ in GaAs will perturb the valence band without affecting mobility. This leads to improvement in speed response. Inter sub-band transition occurred in $\mathrm{GaAsNBi} / \mathrm{GaAs}$ between $\mathrm{HH}$ and $\mathrm{LH}$ sub-bands. Inter sub-band transitions occurring between the $\mathrm{VB}$ and $\mathrm{CB}$ sub-bands could make possible the fabrication of solar cells and lasers operating in the $1.5 \mu \mathrm{m}$ wavelength region.

\section{References}

[1] Usman M, Broderick C A and O'Reilly E P 2013 AIP Conf. Proc. 156621

[2] Xiaoyang M et al 2014 Nanoscale Res. Lett. 9580

[3] Wei P et al 2004 Nucl. Instrum. Methods Phys. Res. B 219671

[4] Kini R N, Ptak A J, Fluegel B, France R, Reedy R C and Mascarenhas A 2011 Phys. Rev. B 83075307

[5] Broderick C A et al 2013 Semicond. Sci. Technol. 28125025

[6] Sweeney S J and Jin S R 2013 J. Appl. Phys. 113043110

[7] Yoshimoto M et al 2004 Jpn. J. Appl. Phys. 43 L845

[8] Wei H et al 2005 J. Appl. Phys. 98053505

[9] Lin K I, Lin K L, Wang B W, Lin H and Hwang J S 2013 Appl. Phys. Express 6121202
[10] Samajdar D P et al 2016 Curr. Appl. Phys. 161687

[11] Aissat A et al 2015 Mater. Sci. Semicond. Process. 31568

[12] Fluegel B et al 2006 Phys. Rev.Lett. 97067205

[13] Alberi K et al 2007 Phys. Rev. B 75045203

[14] Habchi M, Nasr A B, Rebey A and Jani B E 2013 Infrared Phys. Technol. 6188

[15] Ng S T, Fan W J, Dang Y X and Yoon S F 2005 Phys. Rev. B 72115341

[16] Vurgaftman I, Meyer J R and Ram-Mohan L R $2001 \mathrm{~J}$. Appl. Phys. 895815

[17] Voon L Y and Willatzen M 2009 The kp method: electronic properties of semiconductors (Berlin Heidelberg: SpringerVerlag)

[18] Mal I et al 2017 Superlattices Microstruct. 10620

[19] Mal I et al 2017 Superlattices Microstruct. 109442

[20] Alberi K, Dubon O D et al 2007 Appl. Phys. Lett. 91 051909

[21] Rajpalke M K, Linhart W M, Birkett M, Yu K M, Scanlon D O, Buckeridge J et al 2013 Appl. Phys. Lett. 103142106

[22] Gladysiewicz M, Kudrawiec R and Wartak M S J 2015 Appl. Phys. 118055702

[23] Pidgeon C R and Brown R N 1966 Phys. Rev. 146575

[24] Bir G L and Pikus G E 1976 Symmetry and strain-induced effects in semiconductors (New York: Wiley)

[25] Bousbih F, Bouzid S B, Chtourou R, Charfi F F, Harmand J C and Ungaro G 2002 Mater. Sci. Eng., C 21251

[26] Chuang S L 1995 Physics of optoelectronic devices (pure and applied optics) (New York: Wiley)

[27] Chow W W and Koch S W 1999 Semiconductor-laser fundamentals: physics of the gain materials (New York: Springer)

[28] Ahmed A B, Saidi H, Ridene S and Bouchriha H 2015 IEEE J. Quantum Electron. 511

[29] Debbichi M et al 2009 Semicond. Sci. Technol. 24085010

[30] Lysak V V, Kawaguchi H and Sukhoivanov I A 2005 IEEE Proc. Optoelectron. 152131

[31] Asada M, Kameyama A and Suematsu Y 1984 IEEE J. Quantum Electron. 20745

[32] Habchi M M, Nasr A B, Rebey A and EI Jani B 2013 Infrared Phys. Technol. 6188

[33] Pan J L and Fonstad C G 2000 IEEE Trans. Electron. Dev. 47 1325

[34] Yoshimoto M et al 2006 Phys. Status Solidi 2431421

[35] Nacer S et al 2008 Opt. Quantum Electron. 40677 